\title{
Comparación del estado nutricional, consumo habitual de alimentos y hábitos de actividad física en escolares de primero básico -primero medio de la ciudad de Taltal, Chile
}

\author{
Comparison of nutritional status, habitual food consumption and \\ physical activity habits of schoolchildren in the city of Taltal, Chile
}

\begin{abstract}
RESUMEN
El artículo compara el estado nutricional, consumo de alimentos y hábitos de actividad física en escolares de primer año básico y primer año medio. Método: mediante diseño descriptivo prospectivo, se exploró antropométricamente y encuestó 225 escolares (94 básicos, 131 medios), previo asentimiento y consentimiento informado. Resultados: según IMC, el 53,8\% del total de estudiantes básicos y medios califica fuera del rango normal, a saber: $30,6 \%$ sobrepeso, $19 \%$ obesidad y $4 \%$ obesos severos. En el total de la muestra, la combinación de alimentos desayuno-colaciones de media tarde es alto en grasas, siendo esta combinación mucho mayor en básicos. Las combinaciones de colaciones moderadas en grasa son preferidas por los varones, a diferencia de las mujeres que consumen colaciones altas en grasas. El 68\% de los hombres y mujeres de enseñanza básica realiza actividad física regularmente, en tanto los de media solo alcanzan un 58,2\%. No se halló diferencias significativas en las variables antropométricas y alimentarias entre estudiantes básicos y medios, excepto en la realización de actividad física entre mujeres. Conclusiones: el sobrepeso y obesidad afectan gran parte de la muestra, las preferencias alimentarias tienen alto contenido graso y la actividad física que realizan decrece desde los estudiantes básicos a los medios.

Palabras clave: Comportamiento escolar; Estado nutricional; Obesidad; Sobrepeso; Valoración antropométrica.
\end{abstract}

ABSTRACT
The article compares the nutritional status, food consumption
and physical activity habits in students. Method: Using a
prospective descriptive design, anthropometric exploration
was conducted and 225 schoolchildren were surveyed (94
primary and 131 high school students), after assent and
informed consent. Results: Over half (53.8\%) had a BMI
outside the normal range, namely: 30.6\% overweight, $19 \%$
obesity and $4 \%$ severe obese. The combination of breakfast
and tea time foods was high in fats. This combination being
much higher in primary school students. Moderate fat snack
combinations were preferred by males, unlike women who
consumed high fat snacks. Among primary school children,

Monserrat Rivera ${ }^{1 *}$, Guido Solari ${ }^{1}$, Bruno Solari², Ana Wall', María Peralta ${ }^{1}$.

\author{
1. Facultad Cs. de la Salud, Universidad de Antofagasta, \\ Antofagasta, Chile. \\ 2. Servicio de Salud, Antofagasta, Chile.
}

\begin{abstract}
*Dirigir correspondencia a: Monserrat Rivera I. Facultad de Cs. de la Salud, Universidad de Antofagasta, Avda. Angamos 601, Teléfono: 999117956. E-mail: monserrat.rivera@uantof.cl
\end{abstract}

Este trabajo fue recibido el 13 de junio de 2019. Aceptado con modificaciones: 22 de agosto de 2019. Aceptado para ser publicado: 01 de octubre de 2019.

$68 \%$ performed regular physical activity, compared to $58.2 \%$ of high school students. No significant differences were found in the anthropometric and eating habits variables between primary and high school students, except in physical activity practice among women. Conclusions: Overweight and obesity affected a large part of the sample. We observed a preference for foods with a high fat content and physical activity decreased between primary and high school. Keywords: Anthropometric assessment; Nutritional status; Obesity; Overweight; School behavior.

\section{INTRODUCCIÓN}

El sobrepeso y obesidad constituyen problemas de afectación mundial, la Organización Mundial de la Salud 
reportó el año 2012 que aproximadamente 44 millones de niños menores de 5 años tenía sobrepeso u obesidad y a1 año 1990 alcanzaban 31 millones (OMS 2014).

La encuesta nacional de salud chilena -años 20162017- describió que el 40,8 \% de los adolescentes tenía sobrepeso- obesidad vinculados a la alimentación y sedentarismo, alcanzando el año 2017 un 73\% (MINSAL 2017). Castro y cols. reportaron el año 2018 que, en Santiago de Chile, los índices de sobrepeso y obesidad en escolares entre 7 a 15 años eran similares al promedio de los habitantes del país, y que los estudiantes hombres presentaban mayor prevalencia de obesidad según índice de masa corporal (IMC) 1 .

La relación entre la edad, sobrepeso y obesidad en Chile, fue estudiada por Martínez y cols. ${ }^{2}$ identificando una correlación positiva en la que el sobrepeso-obesidad se incrementaba con la edad escolar teniendo en consideración variables como la cantidad y calidad de la ingesta alimentaria, actividad física y sexo. Estudios como los de Chacón y colaboradores publicados el año 2015, reportaron hombres con mayor riesgo de obesidad.

Importante es la responsabilidad de los padres o apoderados como proveedores de alimentos, quiénes a pesar de la información disponible en los medios, dotan a los menores de alimentos extras y colaciones con alto contenido graso como galletas y bebidas azucaradas ${ }^{3}$ entre otros. Datos similares han sido publicados en un estudio utilizando una muestra de adolescentes escolares de Brasil, concluyendo que la mitad de ellos consumía semanalmente colaciones basadas en galletas y salados varios ${ }^{4}$. Los ejemplos sobre esta materia son abundantes en las referencias científicas.

Los adolescentes por lo general pueden adquirir sus propios alimentos, no obstante, prefieren aquellos con alto contenido graso y azucarados con mayor riesgo dietético, qué asociado al incremento de la inactividad física, se expresa en ganancia de peso corporal ${ }^{5}$ e incremento del IMC.

En materia de actividad física, se ha reportado que con el incremento de la edad escolar se optimiza la condición física $^{6}$. Algunos autores han reportado que dicho incremento evoluciona con el deterioro de la condición física ${ }^{7,8,9}$. El tema es controversial y en Chile se vincula a la zona geográfica y nivel socioeconómico.

El incentivo de la práctica de la actividad física se relaciona con las actividades programáticas provistas por el colegio y la realización de actividades deportivas de los infantes ${ }^{10}$ incentivados por sus padres. El presente artículo aporta a la identificación del estado nutricional, consumo habitual de alimentos y hábitos de actividad física en escolares de primer año básico y primer año medio, reconoce sus características y provee información basada en las características de la propia población estudiada, para el control de la obesidad y sobrepeso de niños y jóvenes en edad escolar.

\section{MATERIALES Y MÉTODOS}

El estudio reclutó una muestra de alumnos regulares de todas las escuelas públicas y liceos existentes de la comuna de Taltal, conformada por estudiantes de $1^{\circ}$ básico y de $1^{\circ}$ medio, que en total constituían 225 escolares, 94 de enseñanza básica (50 hombres y 44 mujeres) y 131 de enseñanza media (71 hombres y 60 mujeres) (Tabla 1).

Mediante un diseño descriptivo y prospectivo se exploró en cada estudiante algunas variables antropométricas, las características del consumo de alimentos y sus hábitos de actividad física. Los datos fueron recolectados en las instalaciones de los propios centros educativos, en los horarios escolares regulares.

El peso y la estatura de los estudiantes fueron determinados por nutricionistas entrenadas que utilizaron una báscula de columna marca Seca 220, un tallímetro portátil marca Seca 213 y una cinta métrica metálica marca Cescorf para medir el perímetro abdominal (PA) o perímetro de cintura (PC).

Se identificó el IMC y en materia de estado nutricional, se comparó dicho índice de cada estudiante según los puntos de corte propuestos por el Ministerio de Salud de Chile acorde a la norma para evaluación nutricional de niños-niñas y adolescentes de 5 a 19 años de edad ${ }^{11,12}$ que menciona 6 diagnósticos nutricionales con sus respectivas desviaciones estándares, a saber: Obesidad severa: $>+3$; Obesidad: $>+2$ a $+2,9$; Sobrepeso: $>1$ a 1,9; Eutrofia: $+0,9$ a -0,9; Bajo peso: $<-1$ a -1,9; Desnutrición: $<-2$.

Para estimar el consumo de alimentos, nutricionistas capacitadas aplicaron a los padres de los estudiantes de $1^{\circ}$ básico y a los estudiantes de $1^{\circ}$ medio, una encuesta de hábitos de ingesta alimentaria utilizada y publicada previamente por otros autores, para explorar el número de comidas diarias y las características cualitativas de los desayunos, almuerzos, colaciones de media tarde, colaciones $y$ alimentos extras ${ }^{13}$.

Para recopilar información confiable sobre la cantidad de alimentos consumidos por los estudiantes, los encuestadores utilizaron un libro con imágenes de distintas porciones de alimentos, con transformación a gramos validada para escolares chilenos. En el caso de los alimentos envasados, los encuestadores consultaron por la marca del producto para obtener la cantidad directamente del envase.

Para estimar los hábitos de actividad física, se consultó a los padres, tutores y estudiantes mediante una encuesta utilizada en una publicación similar a la presente, para evaluar el tipo de actividad física, las actividades de gasto mínimo, el total de cuadras caminadas, los juegos recreativos y las actividades físicas sistemáticas, asignando un puntaje de acorde con la cuantificación de cada una de las actividades ${ }^{13,14}$.

Los datos, fueron procesados empleando el programa estadístico SPSS considerando la descripción y el análisis estadístico de las variables. Se determinó cálculo de $p$ según estado nutricional, combinaciones de alimentos, colaciones, alimentos extras y actividad física según sexo y nivel escolares, se aplicó la prueba de hipótesis de diferencias de proporciones binomiales, usando el estadístico $Z$ y un nivel de confianza de $95 \%$. 
Previo a la toma de datos, el presente estudio fue sometido a la evaluación ética y metodológica del comité de ética acreditado de la Universidad de Antofagasta (CEIC-UA) de modo que a los padres, apoderados o tutores legales y estudiantes de $1^{\circ}$ medio, se solicitó el consentimiento escrito que informaba a cerca del objetivo del estudio, descripción de las evaluaciones que serían objeto, como a si mismo de su participación como informante de las encuestas. Respecto de los estudiantes básicos, se les explicó verbalmente los procedimientos de evaluación y solo una vez aceptados el asentimiento del menor y el consentimiento de sus padres o tutores, se procedió a la inclusión de cada uno de ellos como parte del estudio.

\section{RESULTADOS}

Según sexo, las evaluaciones antropométricas tendieron a agruparse normalmente con desviaciones estándares (DE) menores. Solo los valores promedios de perímetro de cintura y peso corporal de los estudiantes de primero medio se agruparon con alta DE (Tabla 1).

$\mathrm{Al}$ agrupar las muestras, considerando la clasificación del IMC según el sobrepeso, obesidad y obesidad severa, se observó que el 53,8\% del total de la muestra se ubicó fuera del rango de normalidad y se agrupó según 30,6\% (sobrepeso), 19,1\% (obesos) y $4 \%$ (obesos severos).

Al comparar los grupos de $1^{\circ}$ básico y de $1^{\circ}$ medio, se observó que los varones de básica presentaron mayor peso corporal y que el $66 \%$ de ellos se agrupó fuera del rango de la normalidad. En la misma materia, los varones de enseñanza media tuvieron porcentajes más bajos (49\%) de anormalidad. Por su parte, las mujeres de enseñanza básica y media presentaron similares resultados de peso corporal, registrando ambos grupos, importancias relativas fuera de los rangos de normalidad ( $50 \%$ y $51 \%$ respectivamente) (Tabla 2 ).

Al observar el tipo de ingesta como las combinaciones de desayuno-colación de media tarde, la conducta fue similar en estudiantes básicos y en medios, a saber, ambos grupos -independiente del sexo- presentaron preferencias por alimentos con alto contenido de grasas. Respecto de la misma materia, los estudiantes básicos se agruparon en la combinación de alimentos con Alto contenido de grasa, alcanzando un $70 \%$.

Tabla 1. Distribución de media, desviación estándar (DE), varianza y valores mínimos y máximos de la edad, talla, perímetro de cintura, peso corporal e IMC según sexo y nivel de escolaridad.

\begin{tabular}{|c|c|c|c|c|c|c|c|}
\hline Nivel & Sexo & Variable & Media & $\begin{array}{l}\text { Desviación } \\
\text { estándar } \\
\text { (DE) }\end{array}$ & Varianza & $\begin{array}{c}\text { Valor } \\
\text { mínimo }\end{array}$ & $\begin{array}{l}\text { Valor } \\
\text { máximo }\end{array}$ \\
\hline \multirow{10}{*}{$1^{\circ}$ Básico } & \multirow{5}{*}{$\begin{array}{l}\text { Mujeres } \\
\text { (n 44) }\end{array}$} & Edad (años) & 6,52 & 0,530 & 0,286 & 6 & 8 \\
\hline & & Talla $(\mathrm{cm})$ & 121 & 5,57 & 31,9 & 110 & 133 \\
\hline & & Perímetro de cintura (c) & 58,6 & 5,18 & 26,9 & 48 & 73 \\
\hline & & Peso corporal (kg) & 26,2 & 4,16 & 17,9 & 20 & 37 \\
\hline & & IMC (kg/talla $\left.{ }^{2}\right)$ & 17,7 & 2,09 & 4,38 & 14,5 & 23,5 \\
\hline & \multirow{5}{*}{$\begin{array}{l}\text { Hombres } \\
\text { (n 50) }\end{array}$} & Edad (años) & 6,45 & 0,480 & 0,234 & 6 & 7,11 \\
\hline & & Talla (c) & 120 & 4,79 & 22,9 & 109 & 130 \\
\hline & & Perímetro de cintura(c) & 59,0 & 6,18 & 38,3 & 50 & 80 \\
\hline & & Peso corporal $(\mathrm{kg})$ & 26,3 & 4,88 & 23,9 & 19,5 & 45,5 \\
\hline & & IMC (kg/talla $\left.{ }^{2}\right)$ & 17,9 & 2,41 & 5,85 & 14,3 & 26,7 \\
\hline \multirow{10}{*}{$1^{\circ}$ Medio } & \multirow{5}{*}{$\begin{array}{c}\text { Mujeres } \\
\text { (n 60) }\end{array}$} & Edad (años) & 14,9 & 0,840 & 0,712 & 14 & 17,0 \\
\hline & & Talla $(\mathrm{cm})$ & 158 & 6,19 & 38,5 & 145 & 173 \\
\hline & & Perímetro de cintura (c) & 75,9 & 10,3 & 106 & 59,0 & 102 \\
\hline & & Peso corporal (kg) & 62,9 & 14,1 & 199 & 38,0 & 114 \\
\hline & & IMC (kg/talla $\left.{ }^{2}\right)$ & 24,9 & 5,09 & 25,9 & 15,8 & 40,2 \\
\hline & \multirow{5}{*}{$\begin{array}{c}\text { Hombres } \\
(\mathrm{n} 71)\end{array}$} & Edad (años) & 14,9 & 0,861 & 0,742 & 14 & 19,1 \\
\hline & & Talla $(\mathrm{c})$ & 167 & 6,79 & 46,3 & 144 & 189 \\
\hline & & Perímetro de cintura(c) & 77,4 & 10,1 & 102 & 62,8 & 105 \\
\hline & & Peso corporal $(\mathrm{kg})$ & 67,2 & 13,8 & 190 & 46,5 & 101 \\
\hline & & IMC (kg/talla $\left.{ }^{2}\right)$ & 23,9 & 4,51 & 20,4 & 17,3 & 35,9 \\
\hline
\end{tabular}


Tabla 2. Distribución estado nutricional por el indicador índice de masa corporal, según sexo y nivel de escolaridad.

\begin{tabular}{|c|c|c|c|c|c|c|c|c|c|c|}
\hline \multirow[t]{3}{*}{ Clasificación } & \multicolumn{2}{|c|}{ Básica } & \multicolumn{6}{|c|}{ Media } & \multirow{3}{*}{$\begin{array}{l}\text { Valor P } \\
\text { hombres }\end{array}$} & \multirow{2}{*}{$\begin{array}{l}\text { Valor P } \\
\text { Mujeres }\end{array}$} \\
\hline & \multicolumn{2}{|c|}{$\begin{array}{c}\text { Hombres } \\
n=50\end{array}$} & \multicolumn{2}{|c|}{$\begin{array}{c}\text { Mujeres } \\
n=44\end{array}$} & \multicolumn{2}{|c|}{$\begin{array}{c}\text { Hombres } \\
n=71\end{array}$} & \multicolumn{2}{|c|}{$\begin{array}{c}\text { Mujeres } \\
n=60\end{array}$} & & \\
\hline & $\mathrm{N}$ & $\%$ & $\mathrm{~N}$ & $\%$ & $\mathrm{~N}$ & $\%$ & $\mathrm{~N}$ & $\%$ & & \\
\hline Obesidad severa & 4 & 8 & 1 & 2,3 & 2 & 2,8 & 2 & 3,3 & 0,194 & 0,763 \\
\hline Obesidad & 16 & 32 & 6 & 13,6 & 10 & 14,1 & 11 & 18,3 & 0,182 & 0,521 \\
\hline Sobrepeso & 13 & 26 & 15 & 32,1 & 23 & 32,3 & 18 & 30 & 0,455 & 0,818 \\
\hline Eutrofia & 17 & 34 & 21 & 47,7 & 32 & 45,1 & 27 & 45 & 0,220 & 0,784 \\
\hline Bajo peso & 0 & 0 & 1 & 2,3 & 4 & 5,6 & 1 & 1,6 & 0,088 & 0,399 \\
\hline Desnutrición & 0 & 0 & 0 & 0 & 0 & 0 & 1 & 1,6 & 0 & 0 \\
\hline
\end{tabular}

*Valor de $\mathrm{p}$ en la comparación de la distribución del estado nutricional según IMC, por sexo y nivel educacional, mediante prueba de hipótesis de diferencia de proporciones binomiales, con un 95\% de confianza.

En el caso de las combinaciones Almuerzo-comida, todos los grupos independientemente del sexo y del nivel de escolaridad, presentaron preferencia por alimentos Moderados en grasas alcanzando cada grupo valores cercanos al 55\% (Tabla 3).

En las combinaciones de colaciones, los varones, de básica y media, prefirieron alimentos moderados en grasas, a diferencia de las mujeres de ambos niveles, quienes manifestaron su preferencia por colaciones con alto contenido de grasa.

No obstante, al ordenar toda la muestra de estudiantes según preferencia de alimentos extras, los hombres y las mujeres de básica y de media, se agruparon en los alimentos con alto contenido graso, siendo las mujeres de media

Tabla 3. Distribución de los estudiantes según combinaciones de alimentos por tipo de comida, sexo y nivel de escolaridad.

\begin{tabular}{|c|c|c|c|c|c|c|c|c|c|c|c|c|}
\hline \multirow{4}{*}{ Combinaciones } & \multirow{3}{*}{\multicolumn{2}{|c|}{$\begin{array}{c}\text { Básica } \\
\begin{array}{c}\text { Hombres } \\
(\mathbf{n}=\mathbf{5 0}) \\
\text { *D-O }{ }^{* * A} \text { A-C }\end{array}\end{array}$}} & \multicolumn{6}{|c|}{ Media } & \multirow{2}{*}{\multicolumn{2}{|c|}{$\begin{array}{c}\text { Valor } \mathrm{p}^{*} \\
\text { Hombres }\end{array}$}} & \multirow{2}{*}{\multicolumn{2}{|c|}{$\begin{array}{c}\text { Valor } \mathrm{p}^{* *} \\
\text { Mujeres }\end{array}$}} \\
\hline & & & \multirow{2}{*}{\multicolumn{2}{|c|}{$\begin{array}{c}\text { Mujeres } \\
(\mathbf{n = 4 4 )} \\
* \text { D-O **A-C }\end{array}$}} & \multirow{2}{*}{\multicolumn{2}{|c|}{$\begin{array}{c}\text { Hombres } \\
(\mathbf{n}=71) \\
* \mathrm{D}-\mathrm{O}{ }^{* * A-C}\end{array}$}} & \multirow{2}{*}{\multicolumn{2}{|c|}{$\begin{array}{c}\text { Mujeres } \\
(\mathbf{n}=60) \\
* \text { D-O } * * A-C\end{array}$}} & & & & \\
\hline & & & & & & & & & $* \mathrm{D}-\mathrm{O}$ & ${ }^{* *} \mathrm{~A}-\mathrm{C}$ & $* \mathrm{D}-\mathrm{O}$ & ${ }^{* *} \mathrm{~A}-\mathrm{C}$ \\
\hline & $\%$ & $\%$ & $\%$ & $\%$ & $\%$ & $\%$ & $\%$ & $\%$ & & & & \\
\hline $\begin{array}{l}\text { Alto en } \\
\text { grasas }\end{array}$ & 70 & 26 & 70,5 & 27,2 & 43,6 & 18,3 & 48,3 & 16,6 & 0,004 & 0,023 & 0,023 & 0,190 \\
\hline $\begin{array}{l}\text { Moderado } \\
\text { en grasas }\end{array}$ & 18 & 58 & 25 & 54,5 & 30,9 & 59,1 & 38,3 & 50 & 0,109 & 0,903 & 0,146 & 0,649 \\
\hline $\begin{array}{l}\text { Bajo en } \\
\text { grasas }\end{array}$ & 12 & 16 & 4,5 & 18,1 & 25,3 & 22,5 & 13,3 & 33,3 & 0,070 & 0,377 & 0,131 & 0,084 \\
\hline
\end{tabular}

D-O: combinación desayuno-colación de media tarde, A-C: combina ción almuerzo-comida.

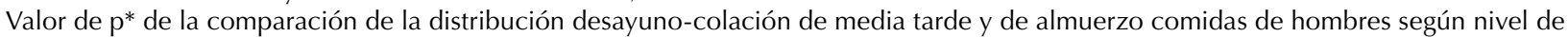
escolaridad mediante la prueba de hipótesis de diferencias de proporciones binomiales, con una confianza del $95 \%$.

Valor de $\mathrm{p}^{* *}$ de la comparación de la distribución desayuno-colación de media tarde y de almuerzo comidas de mujeres según nivel de escolaridad mediante la prueba de hipótesis de diferencias de proporciones binomiales, con una confianza del $95 \%$. 
quienes alcanzaron el más alto valor $(61,5 \%$ del total de su muestra) (Tabla 4).

Respecto de la actividad física, los estudiantes de $1^{\circ}$ medio caminaban mayor número de cuadras. Sin embargo, al considerar el tiempo dedicado a los juegos recreativos diarios y a las actividades sistemáticas, fueron los estudiantes $1^{\circ}$ básico quienes alcanzaron un porcentaje mayor. Al considerar el promedio de la totalidad de las actividades físicas realizadas por todos los estudiantes, fueron las mujeres de $1^{\circ}$ medio quienes realizaban menos actividad física, alcanzando un promedio de 50,6 \% a diferencia de las mujeres de $1^{\circ}$ básico quienes alcanzaron un promedio mayor (70,4\%) (Tabla 5).

Al analizar los resultados entre estudiantes de enseñanza básica y media no se halló diferencias significativas entre ambos grupos para las variables IMC en cada una de sus sub clasificaciones, almuerzo- comida, colaciones y alimentos extras, a diferencia de la variable desayuno -colación de media tarde (tanto en hombres como mujeres) con alto contenido graso, donde si se halló diferencias significativas (Tabla 3). En el caso de la actividad física, al analizar el número de cuadras caminadas entre niveles de escolaridad básicos y medios se observó diferencias, pero estas no fueron estadísticamente significativas. Sin embargo y en la misma materia, pero considerando las actividades físicas de juegos recreativos y actividades físicas sistemáticas, fueron las únicas variables con diferencias significativas entre grupos de mujeres de básica respecto de las mujeres de media.

Tabla 4. Distribución de los estudiantes según colaciones, alimentos extras, sexo y nivel de escolaridad.

\begin{tabular}{|c|c|c|c|c|c|c|c|c|c|c|c|c|}
\hline \multirow{3}{*}{ Combinaciones } & \multicolumn{4}{|c|}{ Básica } & \multicolumn{4}{|c|}{ Media } & \multirow{2}{*}{\multicolumn{2}{|c|}{$\begin{array}{c}\text { Valor } \text { p* }^{*} \\
\text { Hombres }\end{array}$}} & \multirow{2}{*}{\multicolumn{2}{|c|}{$\begin{array}{l}\text { Valor } \mathbf{p}^{* *} \\
\text { Mujeres }\end{array}$}} \\
\hline & \multicolumn{2}{|c|}{$\begin{array}{l}\text { Hombres } \\
\quad(n=50)\end{array}$} & \multicolumn{2}{|c|}{$\begin{array}{r}\text { Mujeres } \\
(n=44)\end{array}$} & \multicolumn{2}{|c|}{$\begin{array}{c}\text { Hombres } \\
\quad(n=71)\end{array}$} & \multicolumn{2}{|c|}{$\begin{array}{r}\text { Mujeres } \\
(n=60)\end{array}$} & & & & \\
\hline & $\begin{array}{c}\text { Col } \\
\%\end{array}$ & $\begin{array}{c}\text { Ext. } \\
\%\end{array}$ & $\begin{array}{c}\text { Col } \\
\%\end{array}$ & $\begin{array}{c}\text { Ext. } \\
\%\end{array}$ & $\begin{array}{c}\text { Col } \\
\%\end{array}$ & $\begin{array}{c}\text { Ext. } \\
\%\end{array}$ & $\begin{array}{l}\text { Col } \\
\%\end{array}$ & $\begin{array}{l}\text { Ext. } \\
\%\end{array}$ & Col & Ext. & Col & Ext. \\
\hline Alto en grasas & 34 & 54 & 40,9 & 52,2 & 33,8 & 60,6 & 48,3 & 61,7 & 0,981 & 0,417 & 0,451 & 0,334 \\
\hline Moderado en grasas & 52 & 28 & 31,8 & 25 & 42,3 & 19,7 & 36,7 & 21,7 & 0,289 & 0,286 & 0,606 & 0,689 \\
\hline Bajo en grasas & 14 & 18 & 27,2 & 22,7 & 23,9 & 19,7 & 15 & 16,7 & 0,176 & 0,013 & 0,125 & 0,439 \\
\hline
\end{tabular}

Col: colaciones, Ext: alimentos extras.

Valor de $\mathrm{p}^{*}$ de la comparación de hombres según nivel escolar de colaciones y alimentos extras, mediante la prueba de hipótesis de diferencias de proporciones binomiales, con una confiabilidad del 95\%.

Valor de $\mathrm{p}^{* *}$ de la comparación de mujeres según nivel escolar de colaciones y alimentos extras, mediante la prueba de hipótesis de diferencias de proporciones binomiales, con una confiabilidad del $95 \%$.

Tabla 5. Distribución de los estudiantes según cuadras caminadas diarias, juegos recreativos diarios, actividad física sistemática semanal según sexo y nivel de escolaridad.

\begin{tabular}{|c|c|c|c|c|c|c|}
\hline Actividad física & \multicolumn{2}{|c|}{\begin{tabular}{cc}
\multicolumn{2}{c}{ Básica } \\
Hombres & Mujeres \\
$(n=50) \quad(n=44)$
\end{tabular}} & $\begin{array}{r}\text { Me } \\
\text { Hombres } \\
(n=71)\end{array}$ & $\begin{array}{l}\text { dia } \\
\text { Mujeres } \\
(n=60)\end{array}$ & $\begin{array}{l}\text { Valor } \mathrm{p}^{*} \\
\text { Hombres }\end{array}$ & $\begin{array}{c}\text { Valor } \mathrm{p}^{* *} \\
\text { Mujeres }\end{array}$ \\
\hline & $\%$ & $\%$ & $\%$ & $\%$ & & \\
\hline $5 y+$ cuadras caminadas diariamente & 44 & 50 & 60 & 53,3 & 0,082 & 0,737 \\
\hline 30 minutos y más de juegos recreativos diarios & 72 & 75 & 61,9 & 50 & 0,247 & 0,009 \\
\hline 2 horas y más de actividad física sistémica semanal & 82 & 86,4 & 76,1 & 48,3 & 0,432 & 0,001 \\
\hline
\end{tabular}

Valor de p* de la comparación de la distribución cuadras caminadas diariamente, juegos recreativos diarios y actividad física sistemática semanal de hombres según nivel de escolaridad, mediante prueba de hipótesis de diferencias de proporciones binomiales, con una confianza del 95\%.

Valor de $\mathrm{p}^{* *}$ de la comparación de la distribución cuadras caminadas diariamente, juegos recreativos diarios y actividad física sistemática semanal de Mujeres según nivel de escolaridad, mediante prueba de hipótesis de diferencias de proporciones binomiales, con una confianza del $95 \%$. 


\section{DISCUSIÓN}

El incremento de la prevalencia del sobrepeso y la obesidad se asocia al deterioro de la condición física en una relación que ha ido evolucionando, pero que ha sido diferente en países según su nivel de desarrollo. Diversas publicaciones reportan estas diferencias comparando muestras diversas. Existen reportes de revisiones sistemáticas que refieren niveles de obesidad y sobrepeso del $40 \%$ de la muestra estudiada ${ }^{15}$. A saber, Herazo y cols. han reportado en Colombia un $42 \%$ de sobrepeso-obesidad en su muestra ${ }^{16}$ a diferencia de nuestros resultados en los que el $54 \%$ del total de nuestra muestra presentó sobrepeso y obesidad. No obstante, las diferencias reportadas, es innegable que la obesidad y el sobrepeso constituyen un problema en aumento que reclama a atención de los estados en consideración a los efectos negativos que tiene en la salud de la población.

Los resultados de nuestro estudio en materia de IMC fueron muy similares a los hallados por Castro y cols, en otra población de escolares chilenos. Ellos reportaron que un $55 \%$ de población estudiada calificaba con sobrepeso u obesidad y que las mujeres estaban más cercanas a la normalidad, a diferencia de nuestro estudio en el que las mujeres -particularmente las de $1^{\circ}$ medio- presentaron un porcentaje levemente superior de IMC que los varones $(51 \% \text { y } 49 \% \text { respectivamente) })^{17}$.

Si consideramos la variable sexo e IMC, nuestro estudio halló que -en promedio-el 57\% de hombres calificaba con sobrepeso, a diferencia de las mujeres que alcanzaban el $50 \%$, estos datos coinciden con los resultados obtenidos por Bazan ${ }^{18}$ y Vasconcelos quienes encontraron que los hombres presentan mayor IMC en relación con las mujeres ${ }^{19}$. Por otra parte, otros estudios realizados en España ${ }^{20}$, han señalado que solo el $22,1 \%$ de la población presentaba exceso de peso según IMC (sobrepeso y obesidad). No obstante, esta condición difiere con otros hallazgos encontrados en Chile en los que el $55,4 \%$ del total de la muestra estudiada calificó con sobrepeso u obesidad y en los que las mujeres presentaban mayor IMC ${ }^{21}$.

En materia de edad, el presente estudio describe que los estudiantes de educación básica tienen mayor IMC de modo similar a lo encontrado por Aguilar y cols, quienes hallaron que, a menor edad, mayor era el sobrepeso u obesidad $^{22,23}$. Sin embargo, existen otros estudios que han planteado resultados diversos y describen que a medida que se incrementa la edad, aumenta el sobrepeso y que las mujeres adolescentes son quienes presentan mayor incremento ${ }^{24}$. En general, en lo relacionado con el IMC, si bien el sobrepeso, obesidad es una realidad nacional acorde a lo encontrado en nuestra investigación, existen algunos estudios ${ }^{25}$ chilenos que no han hallado la misma relación.

En materia de combinaciones de alimentos, nuestro estudio describe que la muestra de estudiantes de educación media y básica prefieren desayunos con alto contenido de grasa y que el incremento de la edad no varía la características del desayuno, a diferencia de lo señalado por algunos autores, que han encontrado que parte importante de los estudiantes -a medida que avanza su edad-omiten el desayuno $\mathrm{o}^{25,26,28}$. No obstante lo anterior, en nuestro estudio, la ingesta diaria de alimentos de todos los estudiantes estudiados, se caracteriza por un alto contenido graso y alimentos ultra procesados, cuestión que se asemeja a lo hallado por Calderón y Garcia ${ }^{29,30}$.

El presente artículo además describe que las altas preferencias por los alimentos extras y colaciones constituyen un hallazgo principalmente asociado a los estudiantes adolescentes en relación con los de básica, como asimismo el alto consumo diario de bebidas azucaradas. Estas preferencias fueron también encontradas por López y cols, quienes concluyeron respecto de la conveniencia de adoptar estrategias de educación nutricional, en razón que los adolescentes tienen mayor autonomía para adquirir alimentos azucarados -en relación con los menores de educación básica- y porque la educación nutricional podría proporcionar un efectivo medio de control para disminuir este tipo de ingesta y con ello, la predisposición al sobrepeso-obesidad ${ }^{31}$.

En materia de actividad física, el presente estudio describe que los estudiantes de $1^{\circ}$ básico tienen mayor actividad mediante juegos recreativos y actividades físicas sistemática y que las mujeres de básica alcanzan los niveles más altos de actividad física de la muestra. Lo anterior no va en la misma dirección de los hallazgos de Martínez y cols. quienes señalan en su estudio que son las mujeres aquellas que prioritariamente realizan menos actividad física ${ }^{32}$.

Según lo reportado por Viera ${ }^{34}$, los estudiantes de la educación media practican menos actividad física que los de básica, respecto de esa misma materia, nosotros encontramos que estos últimos solo incrementan la actividad física diaria, principalmente sobre la base del número de cuadras caminadas, y que son las mujeres adolescentes quienes -en el total de la población estudiada- presentan los niveles más bajos de actividad física (50\%). Estos resultados coinciden con los hallazgos de Medina, quien señala al concluir su estudio, que las mujeres adolescentes podrían ser consideradas físicamente inactivas ${ }^{33}$, a diferencia de lo reportado por Garcés, quien halló mayor actividad física en las mujeres adolescentes que en los hombres ${ }^{35}$.

La disminución del interés por la realización de actividad física a medida que se incrementa la edad en los estudiantes ha sido motivo de investigaciones que han tratado de identificar los factores asociados a dicho desinterés ${ }^{36}$. Según algunos autores, la falta de motivación por el ejercicio o actividad física puede llevar a que los estudiantes ejecuten menos de 4 horas de actividad física semana ${ }^{37}$ que en rigor implica que solo realizan las horas de actividad física obligatorias propuestas por el Ministerio de educación. Nuestro estudio no es muy diferente a lo planteado, de modo que los estudiantes de media reportaron menos actividad física que los de básica de modo similar a los hallazgos de otros autores ${ }^{38,39}$. 


\section{CONCLUSIONES}

La población estudiada padece de alta prevalencia de sobrepeso-obesidad en ambos grupos de estudiantes básicos y medios, con mayor prevalencia en el grupo de $1^{\circ}$ básico. En materia de ingesta diaria de alimentos, ambos grupos tienen notable preferencia por alimentos con alto contenido graso.

En relación con la actividad física realizada, los menores de educación básica dedican más tiempo diario/semanal a la realización de actividad física principalmente lúdica y facilitada por juegos recreativos además de las actividades físicas sistemática. El grupo de adolescentes mujeres de $1^{\circ}$ medio, es el que menos tiempo dedica a la realización de actividades físicas.

A la luz de los resultados, a los autores les parece necesario generar un diseño de intervención inclusivo para controlar el problema estudiado, que incorpore padres, docentes, directivos, estudiantes y representantes de la alcaldía. El diseño de la intervención debe ser diferenciado según sea dirigido a los menores de $1^{\circ}$ básico o a los adolescentes de $1^{\circ}$ medio y estar enfocado en lo cognitivo y lo conductual, implementando desde un inicio la metodología de coaching para instruir y/o entrenar a padres, docentes y estudiantes con el objetivo de conseguir los cambios de conducta en materia de consumo habitual de alimentos y hábitos de actividad física.

\section{BIBLIOGRAFIA}

1. Castro M, Muros J, Cofre C, Zurita F, Chacón R, Espejo T. Rates of overweight and obesity in schoolchildren of Santiago (Chile). J Sport Health Res 2018; 10: 251-256.

2. Vio F. The prevention of obesity in Chile. Rev Chil Nutr 2005; 32: 80-87.

3. Ramírez-Izcoa A, Sánchez-Sierra L, Mejía-Irías C, Izaguirre González A, Alvarado-Avilez C, Flores-Moreno R, Rivera E. Prevalence and factors associated with childhood overweight and obesity in public and private schools in Tegucigalpa, Honduras. Rev Chil Nutr 2017; 44: 161-169.

4. Oliveira S, Bauermann G, Alves M.Toral N. Association between consumption and eating behavior, screen time and physical activity among Brazilian adolescents. Rev Chil Nutr 2018; 45: 349-355.

5. Silva S, Toral N. Profile of the offered in school canteens and their regulation in Brazil. Rev Chil Nutr 2018; 45: 393-399.

6. Moro P, del Castillo, de Espinosa M, Algaba E, López-Ejeda $N$, Serrano M. Semi-longitudinal analysis of physical status in madrilenian adolescents. Br J sport Med 2016; 173: 183-193.

7. Zuñiga $P$, Jaque $B$, González $P$, Faúndez Casanova $C$. Longitudinal study of the nutritional status and physical condition of high school students of a municipal high school in Talca, Chile. Cienc Act Fis UCM 2018; 19: 21-30.

8. Garcinuño A, García I, Alonso I, López S. Determinants of the level of physical activity in school children and adolescents: OPACA study. An Pediatr 2011; 74: 15-24.

9. Delgado-Floody $P$, Caamaño $F$, Osorio A, Jerez D, Fuentes J, Levin E, Tapia J. Body Image and self-esteem in children according to the nutritional status and frecuency of physical activity. Rev Chil Nut 2017; 44: 12-18.

10. Salas C, Petermann-Rocha F, Celis-Morales C, Martínez-López
E. Parental support for physical activity in schoolchildren and its influence on nutritional status and fitness. Rev Chil Pediatr 2018; 89: 732-740.

11. Ministerio de Salud (MINSAL) Division of Healthy Public Policies and Promotion. Department of Nutrition and Food. Chile 2016.

12. Olivares S, Araneda J, Morales G, Leyton B, Bustos N, Hernández M. Oyarzún M. Attitudes of chilean schoolchildren of socioeconomic levels at the beginning of the implementation of the law that regulates the sale and advertising of foods high in critical nutrients. Nutr Hosp. 2017; 34: 431-438.

13. Burrows R, Díaz E, Sciaraffia V, Gattas V, Montoya A, Lera L. Habits of ingestion and physical activity in school children, according to the type of establishment they attend. Rev Med Chile 2008; 136: 53-63.

14. Brandt E, Pacheco I, Ibañez K. Considerations in the habits of feeding and physical activity in students of second basic cycle of Chile. VRev Peru Cienc Act Fis Deporte 2018; 5: 4-4.

15 Antón A, Morales S, Concepción R, Physical activity programs to combat and overweight in adolescents. Rev Cubana Pediatr 2018; 90: 1-12.

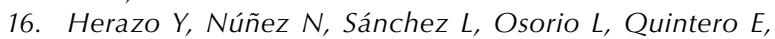
Yepes L, Vázquez K. Physical condition in schoolchildren: differences according to levels of physical activity. Rev Latinoam Hipertension 2018; 13: 317-322.

17. Castro M, Muros J, Cofré C, Zurita F, Chacón R, Espejo T. Overweight and obesity rates in schoolchildren in Santiago (Chile). J Sport Health Res 2018; 10: 251-256.

18. Bazán A, Julia $M$, Sellán $M$, Vázquez A, Díaz M, Domínguez $S$. Factors associated with overweight and childhood obesity in Spain, according to the latest national health survey (2011). Escola Anna Nery 2018; 22: 1-6.

19. Soar C, Vasconcelos F, Assis M. Waist-hip ratio and waist circumference associated with body mass index in a study with schoolchildren. Cad.Saúde Publica 2004; 20: 1609-1616.

20. Prieto D, Correa J, Ramirez R. Levels of physical activity, physical condition and time on screens in schoolchildren in Bogotà, Colombia: study FURECOL. Nutr Hosp.2015; 32: 2184-2192.

21. Floody $P$, Thuillier B, Mayorga $D$, Cofré A, Salazar C. Relation between overweigt obesity, and self-esteem levels in schoolchildren. Retos 2018; 35: 67-70.

22. Aguilar G, Estigarribia G, Sanabria G, Sanabria M, Kawabata A, Munoz S. González C. Overweight, Obesity and fluid intake in children and adolescents in the Capital, Central and Caaguazu, 2016. Pediatría (Asunción) 2018; 45: 147-154.

23. Geserick M, Vogel M, Gausche R, Lipek T, Spielau U, Keller $E$, Körner A. Acceleration of BMI in early childhood and risk of sustained obesity. N Engl J Med 2018; 379: 1303-1312.

24. Shamah T, Cuevas L, Gaona E, Gómez L, Morales M, Hernández M, Rivera J. Overweight and obesity in children and adolescents, 2016 Halfway national health and nutrition survey update. Salud Publica Mex. 2018; 60: 244-253.

25. Rodríguez A, González G, Padilla F. Habits of feeding, physical activity and hours of sleep in schoolchildren: a diagnostic study in Primary Education. Educatio Siglo XXI 2018; 36: 443-464.

26. Ferreruela I, Ruíz C, Piqueres A, Forés Á, Sebastiá N, Cervera M. Analysis of nutritional habits and physical activity in the school population in Benicàssim. Evidentia 2109; 16: 1-6.

27. Díaz T, Ficapa-Cusí P, Aguilar-Martínez A. Breakfast habits in primary and secondary schoolchildren: options for nutritional 
education in schools. Nutr Hosp. 2016; 33: 909-914.

28. Ibarra J, Ventura C, Hernández C. Habits of healthy life of physical activity, feeding, sleep and consumption of tobacco and alcohol, in Chilean teenage students. Sportis 2019; 5: 70-84.

29. Calderón A, Marrodán M, Villarino A, Martínez J. Assessment of the nutritional status and habits and food preferences in a child-youth population ( 7 to 16 years) of the Community of Madrid. Nutr Hosp. 2019; 36: 394-404.

30. Gálvez MI, Cuesta MA, Jiménez EG, Valle JS. Sociodemographic analysis of the state and nutritional level and physical activity of two schools in Granada (Spain). JONNPR. 2017; 2: 152-159.

31. Lopez P, Olivares $R$, Almonacid A, Gomez R, Cossio M, Garcia J. Association between dietary habits and the presence of overweight/obesity in a sample of 21,385 chilean adolescents. Nutr Hosp. 2015; 31: 2088-2094.

32. Martínez J, Borrel V, Reyes M, Pastor J. The Physical education and its repercussion during school recess based on an intervention proposal. A pilot study, ESHPA 2018; 2: 192-206.

33. Medina C, Jáuregui A, Campos I, Barquera S. Prevalence and trends of physical activity in children and adolescents: results of Ensanut 2012 and Ensanut MC 2016. Salud Publica Mex 2018; 60: 263-271.

34. Viera E, Quiñones I, Araujo J, Relationship between physical activity, nutrition and family in school-age youth. Retos 2018; 34: 85-88.

35. Garcés $T$, Ortega $F$, Cuberos $R$, Sánchez $M$, Martínez $A$, Cortés A. Physical activity and self-concept: Study two factors teens of rural área. RIPED 2018; 13: 203-210.

36. Alonso,J Castedo A, Portela I. Validation of the self-report of reasons for the practice of physical exercise with teenagers (AMPEF): differences by gender, age and school year. Retos 2018; 33: 273-278.

37. Castrillo P, Villalaín A, Reboredo B, Valeiro M. Active lifestyles development at school: physical activity, age and gender. Sportis 2019; 5: 53-69.

38. Granizo I, Ortega F, Sánchez M, Cuberos R. Analysis of the motivational climate towards sports and the problematic use of videogames in schoolchildren in Granada. Retos 2019; 35: 255-260.

39. Baena A, Vega $D$, Ramirez J. Predicitve factors of physical activity in Spanish schoolchildren according to their weight status. Retos 2018; 33: 74-78. 